\title{
TESTING FOR UNIT AND FRACTIONAL ORDERS OF INTEGRATION \\ IN THE TREND AND SEASONAL COMPONENTS OF US MONETARY AGGREGATES
}

\author{
Guglielmo Maria Caporale \\ Brunel University, London \\ Luis A. Gil-Alana \\ University of Navarra
}

April 2006

\begin{abstract}
Monthly seasonally unadjusted data can exhibit roots with possibly fractional orders of integration, corresponding to the monthly but also to the quarterly and to the long-run or trending components of the series. In this paper we use a procedure which is suitable to test simultaneously for the order of integration of each of these components and apply it to several US monetary aggregates.
\end{abstract}

JEL Classification: $C 15$, C22

Keywords: Seasonality, Long Memory, Monetary Aggregates

Corresponding author: Professor Guglielmo Maria Caporale, Brunel Business School, Brunel University, Uxbridge, Middlesex UB8 3PH, UK. Tel.: +44 (0)1895 266713. Fax: +44 (0)1895 269770. E-mail: Guglielmo-Maria.Caporale@brunel.ac.uk

The second-named author gratefully acknowledges financial support from the Ministerio de Ciencia y Tecnologia (SEC2005-07657, Spain). 


\section{Introduction}

Modelling macroeconomic time series has received considerable attention in the last few decades. Initially, deterministic approaches were adopted, based on linear (or quadratic) functions of time and/or seasonal dummy variables. These models, however, were shown to be inappropriate in many cases, especially if the trend or the seasonal components changed or evolved over time. Unit root models were then proposed by Box and Jenkins (1970) and test statistics were developed by Dickey and Fuller (1979); these tests became extremely popular, especially after the seminal paper of Nelson and Plosser (1982). Seasonal unit root tests were also proposed for quarterly (e.g. Dickey, Hasza and Fuller, DHF, 1984, and Hylleberg, Engle, Granger and Yoo, HEGY, 1990) and monthly (Hasza and Fuller, HF, 1982, and Beaulieu and Miron, BM, 1993, etc.) data. More recently, the unit root approach has been extended to allow for other types of long-memory behaviour, in particular allowing for fractional degrees of integration in each of the components of the series. A review of fractional processes at the zero frequency is Baillie (1996) and tests of the (possible) fractional order of integration at the seasonal quarterly and monthly components can be found in Gil-Alana and Robinson (2001) and Gil-Alana (1999). These two papers are based on a procedure due Robinson (1994) that is very general in the sense that it allows to consider a large variety of long memory processes.

In this paper, we introduce a version of Robinson's (1994) procedure which is suitable to test simultaneously for the order of integration of the trend and of the seasonal (quarterly and monthly) components of a time series. The tests are fairly general and allow us to test for both unit and fractional degrees of integration. The outline of the paper is as follows: Section 2 describes the version of the tests of Robinson (1994) used in this paper. Section 3 reports finite-sample critical values of the suggested tests, along with some Monte Carlo experiments conducted to examine their size and power properties in finite samples. In 
Section 4, the tests are applied to several US monthly monetary aggregates, while Section 5 contains some concluding comments.

\section{The tests of Robinson (1994)}

Let us assume that $\mathrm{y}_{\mathrm{t}}$ is the time series we observe from $\mathrm{t}=1,2, \ldots, \mathrm{T}$, and consider the following model:

$$
(1-L)^{d_{1}+\theta_{1}}\left(1-L^{4}\right)^{d_{2}+\theta_{2}}\left(1-L^{12}\right)^{d_{3}+\theta_{3}} y_{t}=u_{t}, \quad t=1,2, \ldots,
$$

for given real numbers $d_{1}, d_{2}$ and $d_{3}$, where $L$ and $L^{s}(s=4,12)$ are the lag and the seasonal lag operators $\left(\mathrm{Lx}_{\mathrm{t}}=\mathrm{x}_{\mathrm{t}-1} ; \mathrm{L}^{\mathrm{s}} \mathrm{x}_{\mathrm{t}}=\mathrm{x}_{\mathrm{t}-\mathrm{s}}\right)$ respectively, and $\mathrm{u}_{\mathrm{t}}$ is an $\mathrm{I}(0)$ process, defined as a covariance stationary process with spectral density function, that is positive and finite at any frequency on the interval $[0, \pi]$. Under the null hypothesis, defined by:

$$
\mathrm{H}_{\mathrm{o}}: \theta=\left(\theta_{1} ; \theta_{2} ; \theta_{3}\right)^{\prime}=0
$$

$y_{t}$ in (1) follows a fractionally integrated process with orders of integration: $d_{1}$ (for the long run component); $\mathrm{d}_{2}$ (for the quarterly component); and $\mathrm{d}_{3}$ (for the monthly structure). Robinson (1994) proposed a Lagrange Multiplier (LM) test for testing $\mathrm{H}_{\mathrm{o}}$ (2) in (1) against the alternative $\mathrm{H}_{\mathrm{a}}: \theta \neq 0$. Specifically, the test statistic is then given by:

$$
\hat{\mathrm{R}}=\frac{\mathrm{T}}{\hat{\sigma}^{4}} \hat{\mathrm{a}}^{\prime} \hat{\mathrm{A}}^{-1} \hat{\mathrm{a}}
$$

where

$$
\hat{a}=\left(\hat{a}_{1} ; \hat{a}_{2} ; \hat{a}_{3}\right)^{\prime}, \quad \hat{a}_{i}=\frac{-2 \pi}{T} \sum_{j}^{*} \psi_{i}\left(\lambda_{j}\right) g\left(\lambda_{j} ; \hat{\tau}\right)^{-1} \mathrm{I}\left(\lambda_{j}\right) ;
$$

$\hat{A}$ is a $(3 \times 3)$ matrix with $(\mathrm{r}, \mathrm{s})^{\text {th }}$ element:

$$
\begin{gathered}
\hat{a}_{r s}=\frac{2}{T}\left(\sum_{j}^{*} \psi_{r}\left(\lambda_{j}\right) \psi_{s}\left(\lambda_{j}\right)-\sum_{j}^{*} \psi_{r}\left(\lambda_{j}\right) \hat{\varepsilon}\left(\lambda_{j}\right)^{\prime} x\left(\sum_{j}^{*} \hat{\varepsilon}\left(\lambda_{j}\right) \hat{\varepsilon}\left(\lambda_{j}\right)^{\prime}\right)^{-1} x \sum_{j}^{*} \hat{\varepsilon}\left(\lambda_{j}\right) \psi_{s}\left(\lambda_{j}\right)\right) \\
\psi_{1}\left(\lambda_{j}\right)=\log \left|2 \sin \frac{\lambda_{j}}{2}\right| ; \quad \psi_{2}\left(\lambda_{j}\right)=\psi_{1}\left(\lambda_{j}\right)+\log \left(2 \cos \frac{\lambda_{j}}{2}\right)+\log \left|2 \cos \lambda_{j}\right| ;
\end{gathered}
$$




$$
\begin{gathered}
\psi_{3}\left(\lambda_{j}\right)=\psi_{2}\left(\lambda_{j}\right)+\log \left|2\left(\cos \lambda_{j}-\cos \frac{\pi}{3}\right)\right|+\log \left|2\left(\cos \lambda-\cos \frac{2 \pi}{3}\right)\right|+ \\
\log \left|2\left(\cos \lambda-\cos \frac{\pi}{6}\right)\right|+\log \left|2\left(\cos \lambda-\cos \frac{5 \pi}{6}\right)\right| \\
\hat{\sigma}^{2}=\sigma^{2}(\hat{\tau})=\frac{2 \pi}{T} \sum_{j}^{*} g\left(\lambda_{j} ; \hat{\tau}\right)^{-1} \mathrm{I}\left(\lambda_{j}\right) ; \quad \hat{\varepsilon}\left(\lambda_{j}\right)=\frac{\partial}{\partial \tau} \log g\left(\lambda_{j} ; \hat{\tau}\right) ; \quad \lambda_{j}=\frac{2 \pi j}{T} .
\end{gathered}
$$

The function $\mathrm{g}$ above is known and is obtained from the spectral density function of $\mathrm{u}_{\mathrm{t}}$,

$$
\mathrm{f}(\lambda ; \tau)=\frac{\sigma^{2}}{2 \pi} \mathrm{g}(\lambda ; \tau), \quad-\pi \leq \lambda<\pi
$$

evaluated at $\hat{\tau}=\arg \min _{\tau \in \mathrm{T}} \sigma^{2}(\tau)$. I $\left(\lambda_{\mathrm{j}}\right)$ is the periodogram of $\hat{u}_{t}$, where

$$
\hat{u}_{t}=(1-L)^{d_{1}}\left(1-L^{4}\right)^{d_{2}}\left(1-L^{12}\right)^{d_{3}} y_{t}
$$

and the summation on $*$ in the above expressions are over $\lambda \in M$ where $M=\{\lambda:-\pi<\lambda<\pi$, $\left.\lambda \notin\left(\rho_{1}-\lambda_{1}, \rho_{1}+\lambda_{1}\right), 1=1,2, \ldots, s\right\}$ such that $\rho_{1}, 1=1,2, \ldots, \mathrm{s}<\infty$ are the distinct poles of $\psi(\lambda)$ on $(-\pi, \pi]$

Based on the null hypothesis (2), Robinson (1994) showed that, under certain regularity conditions, ${ }^{1}$

$$
\hat{\mathrm{R}} \rightarrow_{\mathrm{d}} \chi_{3}^{2} \text { as } \mathrm{T} \rightarrow \infty
$$

and, also, the Pitman efficiency property that against local alternatives of the form: $\mathrm{H}_{\mathrm{a}}$ : $\theta=$ $\delta \mathrm{T}^{-1 / 2}$ for $\delta \neq 0, \hat{R}$ has an asymptotic distribution given by $\chi_{3}^{2}(v)$, with a non-centrality parameter, $v$, which is optimal under Gaussianity of $u_{t}$.

Other versions of the tests of Robinson (1994) which analyse separately the orders of integration of each of the components of the series have been used in earlier papers. For example, Gil-Alana and Robinson (1997) consider the case of roots occurring exclusively at the long run or zero frequency in several US macroeconomic historical annual time series, whilst Gil-Alana (1999) and Gil-Alana and Robinson (2001) look respectively at the cases of

\footnotetext{
1 These conditions are very mild regarding technical assumptions to be satisfied by model (1).
} 
roots with monthly and quarterly components. In the present study, we consider another version of Robinson's (1994) testing procedure that allows us to test simultaneously for the orders of integration of each of these components. We then carry out an empirical application using our approach based on fractional integration tests including both trends and seasonal components.

\section{A finite-sample experiment}

This section examines the finite-sample behaviour of the tests described in Section 2 by means of Monte Carlo simulations. Finite-sample critical values of $\hat{R}$ in (3) are computed and their power properties are investigated.

\section{[Insert Table 1 about here]}

Table 1 reports the empirical distribution of $\hat{R}$ in (3) for different sample sizes, $\mathrm{T}=$ 48, 96, 120 and 240, based on 50,000 replications, and compares them to a $\chi_{3}^{2}$ distribution. We assume that $u_{t}$ is a Gaussian white noise process with zero mean and variance 1, generated by the routines GASDEV and RAN3 of Press, Flannery, Teukolsky and Vetterling (1986). One can see that, for practically all the percentiles and all sample sizes, the finitesample critical values are higher than those given by the $\chi_{3}^{2}$ distribution. Thus, when testing $\mathrm{H}_{\mathrm{o}}$ (2) against $\mathrm{H}_{\mathrm{a}}: \theta \neq 0$, the test statistic based on the asymptotic results will reject the null more often than those based on the size-corrected ones. One can also note that, increasing $\mathrm{T}$, the critical values approximate to those given by the $\chi_{3}^{2}$ distribution.

Table 2 reports the rejection frequencies of the tests, based on both the asymptotic and the finite-sample critical values, the null model consisting of three unit roots corresponding to the trend, the seasonal and the monthly components, i.e.,

$$
(1-\mathrm{L})\left(1-\mathrm{L}^{4}\right)\left(1-\mathrm{L}^{12}\right) \mathrm{y}_{\mathrm{t}}=\mathrm{u}_{\mathrm{t}}, \quad \mathrm{t}=1,2, \ldots,
$$

and white noise $\mathrm{u}_{\mathrm{t}}$. The alternatives are such that: 


$$
(1-\mathrm{L})^{1+\theta_{1}}\left(1-\mathrm{L}^{4}\right)^{1+\theta_{2}}\left(1-\mathrm{L}^{12}\right)^{1+\theta_{3}} \mathrm{y}_{\mathrm{t}}=\mathrm{u}_{\mathrm{t}}, \quad \mathrm{t}=1,2, \ldots
$$

with $\theta_{1}, \theta_{2}$, and $\theta_{3}$ equal to $-1,-0.50$ and 0 . Thus, the rejection frequencies corresponding to $\theta_{1}=\theta_{2}=\theta_{3}=0$ will indicate the sizes of the tests. The nominal size is $5 \%$ in all cases.

\section{[Insert Table 2 about here]}

Table 2 shows that the sizes based on the asymptotic results are too large in all cases, although they improve with $\mathrm{T}$. The larger sizes of the asymptotic tests are also associated with some superior rejection frequencies compared with the finite-sample ones. As expected, the lowest probabilities are obtained with $\mathrm{T}=48$, and they considerably improve as the number of observations increases. The lowest value occurs when $\theta_{1}=\theta_{3}=0$ and $\theta_{2}=-0.50$ $(\mathrm{T}=48)$, with a rejection frequency of 0.119 with the finite-sample critical values and 0.224 with the asymptotic results. When increasing the sample size, these values also increase, and they become 0.316 and 0.417 with $\mathrm{T}=96 ; 0.450$ and 0.547 with $\mathrm{T}=120$, and 0.967 and 0.973 with $\mathrm{T}=240$. Finally, when $\mathrm{T}=240$, the difference between the finite-sample and the asymptotic results becomes negligible.

\section{An empirical application}

The version of the tests of Robinson (1994) described in Section 2 is applied in this section to several US monetary aggregates. The data correspond to monthly non-seasonally adjusted observations of the M1, M2, M3 and MZM stocks for the time period 1980m1-1999m12, obtained from the Federal Reserve Bank of St. Louis.

Denoting each series by $\mathrm{y}_{\mathrm{t}}$, we employ throughout model (1), testing $\mathrm{H}_{\mathrm{o}}$ (2) for values of $d_{1}, d_{2}$ and $d_{3}$ equal to $0,0.25,0.50,0.75$ and 1 . Thus, we test for the presence of three unit roots corresponding to the trend, the quarterly and the monthly components (if $\mathrm{d}_{1}=\mathrm{d}_{2}=\mathrm{d}_{3}=$ 1); two unit roots (if, for example, $d_{1}=d_{2}=1$ and $d_{3}=0$ ); a single unit root if one of the $d$ 's 
is equal to 1 and the other two are 0 , as well as other fractionally integrated alternatives for each of the components of the series.

\section{[Insert Table 3 about here]}

Starting with M1, one can see in Table 3 that $H_{o}(2)$ cannot be rejected if $d_{1}=1, d_{2}=$ 0 and $d_{3}=0.25,0.50$ and 0.75 , being rejected for the remaining cases. These results indicate that a unit root appears at the long run or zero frequency, along with a fractional one corresponding to the seasonal monthly component. This fractional structure seems to be crucial to account for the behaviour of this series, given that the integer numbers $\left(\mathrm{d}_{3}=0\right.$ and 1) both result in a rejection. Also, the lowest statistic is obtained when $d_{1}=1, d_{2}=0$ and $d_{3}=$ 0.25 , suggesting that a possible way of modelling this series might be:

$$
(1-\mathrm{L})\left(1-\mathrm{L}^{12}\right)^{0.25} \mathrm{y}_{\mathrm{t}}=\mathrm{u}_{\mathrm{t}}, \quad \mathrm{t}=1,2, \ldots,
$$

with white noise $\mathrm{u}_{\mathrm{t}}{ }^{2}$

\section{[Insert Tables 4 - 6 about here]}

Tables $4-6$ report the same statistic as in Table 3 for the monetary aggregates M2, M3 and MZM respectively. Similarly to the M1 aggregate, the non-rejection values always occur when $d_{1}=1$ and $d_{2}=0$. However, unlike in the previous Table, the values of $d_{3}$ now range between 0 and 0.50 , indicating that the order of integration of the monthly component in these aggregates is smaller by about 0.25 compared to M1. Further, for the aggregates M2 and M3 the lowest statistics are obtained when $d_{3}=0$, suggesting that a random walk $\left(d_{1}=1\right.$, $\mathrm{d}_{2}=\mathrm{d}_{3}=0$ ) may be a plausible way of modelling these series, while for the MZM aggregate a model like (5) could be more appropriate in view of the fact that it has the lowest statistic. ${ }^{3}$

The results in Tables 3 - 6 show that there are very few non-rejection values, which may reflect unaccounted-for $\mathrm{I}(0)$ autocorrelation in $\mathrm{u}_{\mathrm{t}}$. Therefore, we also calculated the test

\footnotetext{
2 Weakly parametrically autocorrelated disturbances will be allowed at the end of this section.

3 Note that the estimates obtained through the lowest statistic should be an approximation to the maximum likelihood estimates since the tests of Robinson (1994) are based on the Whittle function, which is an approximation to the likelihood function.
} 
statistics imposing an autoregressive (AR) structure on the disturbances $\mathrm{u}_{\mathrm{t}}$. We examined the cases of non-seasonal and seasonal AR processes of the form:

$$
u_{t}=\sum_{j=1}^{q} \tau_{j} u_{t-j s}+\varepsilon_{t}, \quad t=1,2, \ldots
$$

with $\mathrm{s}=1,4$ and 12 , and $\mathrm{q}=1$ and 2, for the four monetary aggregates. We do not report all the results here but only those corresponding to $\mathrm{M} 2$ with $\mathrm{q}=1$, and $\mathrm{s}=1$ and 12 . The quarterly seasonal AR model $(s=4)$ produced rejections in all cases, suggesting that the seasonal quarterly component may not be required in this context, thereby corroborating the finding in Tables $3-6$ that $d_{2}$ should be equal to 0 .

Table 7 reports values of $\hat{R}$ in (3) in a model given by (1) with non-seasonal AR(1) disturbances. One can see that, if $d_{1}=0, H_{o}(2)$ cannot be rejected when $d_{2}=0$ and $d_{3}=0$, $0.25,0.50$ and 0.75 , suggesting that the AR coefficient may be picking up the nonstationary character of the trending component of the series. Apart from these cases, all the remaining non-rejection values occur when $d_{2}=0$, with $d_{1}=0.50$ and $d_{3}=0.75 ; d_{1}=0.75$ and $d_{3}=0.25$ and 0.50; and $\mathrm{d}_{1}=1$ and $\mathrm{d}_{3}=0,0.25$ and 0.50 . Thus, it seems that there is some kind of competition between the order of integration at the zero frequency and the AR coefficients in describing the nonstationary nature of the trend component. (Note that we use Yule-Walker estimates for the AR structure, which entails AR roots that are automatically less than one in absolute value, but can be arbitrarily close to one).

\section{[Insert Tables 7 and 8 about here]}

Finally, in Table 8, we report the values of the test statistic when $u_{t}$ in (1) is modelled in terms of a seasonal monthly $\mathrm{AR}(1)$ process. The results are very similar to those reported in Table 4 for the case of white noise disturbances, showing that $\mathrm{H}_{\mathrm{o}}$ (2) cannot be rejected when $d_{1}=1, d_{2}=0$ and $d_{3}$ ranging between 0 and 0.75 . Thus, we can conclude that the inclusion of a seasonal monthly AR process for the disturbances does not eliminate either the 
unit root behaviour of the trending component, or the potential fractional degree of integration of the monthly dynamic structure.

The fact that in most cases $d_{1}$ is close to 1 while $d_{3}$ (the order of integration at the monthly frequency) is strictly smaller than 1 suggests that shocks to US monetary aggregates affecting the long run structure are of a permanent nature. The implication is that policy measures are required to bring monetary aggregates back to their original level. On the other hand, shocks affecting the monthly component appear to be mean-reverting, implying that the effect on monetary aggregates is only temporary, and that these will eventually readjust to their long-run value even without policy intervention.

\section{Conclusions}

In this paper we have introduced a version of the tests of Robinson (1994) for simultaneously testing the orders of integration in the trend and the seasonal quarterly and monthly components of time series. The tests have standard null and local limit distributions. Finitesample critical values were computed and results based on Monte Carlo simulations showed that they perform relatively well against both local and non-local departures from the null. The tests were applied to monthly non-seasonally adjusted data on four US monetary aggregates, and the results indicate that all series contain a unit root at the zero frequency, along with possibly fractional values for the monthly component.

The present study could be extended in several ways. For instance, the AR structure for the $\mathrm{I}(0)$ disturbances could be replaced by other less conventional forms of $\mathrm{I}(0)$ processes, such as the Bloomfield (1973) exponential spectral model. This model has exponentially decaying autocorrelations like the stationary AR model, and it is very easy to compute in the context of the present tests. Also, it would be of interest to obtain point estimates of the orders of integration for each of the components of the series. Note that the approach used here generates diagnostics for departures from real orders of integration, and hence it is not 
surprising that different specifications may not be rejected. Ooms (1995) suggests Wald tests based on Robinson's (1994) model, using for the estimation a modified periodogram regression procedure due to Hassler (1994), whose distributions is obtained using simulation techniques. Similar methods based on this and other semi-parametric procedures (e.g. Robinson, 1995a, b) can also be applied to monetary aggregates or to other macroeconomic time series. 


\section{References}

Baillie, R.T. (1996) Long memory processes and fractional integration in econometrics, Journal of Econometrics, 73, pp. 5-59.

Beaulieu, J.J. \& Miron, J.A. (1993) Seasonal unit roots in aggregate U.S. data, Journal of Econometrics, 55, pp. 305-328.

Bloomfield, P.J. (1973) An exponential model for the spectrum of a scalar time series, Biometrika, 60, pp. 217-226.

Box, G.E.P. \& Jenkins, G.M. (1970) Time series analysis, forecasting and control, San Francisco, Holden-Day.

Dickey, D.A. \& Fuller, W.A. (1979) Distributions for the estimators of autoregressive time series with a unit root, Journal of the American Statistical Association, 74, pp. 427-431.

Dickey, D.A., Hasza, D.P. \& Fuller, W.A. (1984) Testing for unit roots in seasonal time series, Journal of the American Statistical Association, 79, pp. 355-367.

Gil-Alana, L.A. (1999) Testing fractional integration with monthly data, Economic Modelling, 16, pp. 613-629.

Gil-Alana, L.A. \& Robinson, P.M. (1997) Testing of unit roots and other nonstationary hypotheses in macroeconomic time series, Journal of Econometrics, 80, pp. 241-268.

Gil-Alana, L.A. \& Robinson, P.M. (2001) Testing seasonal fractional integration in UK and Japanese consumption and income, Journal of Applied Econometrics 16, 95-114.

Hassler, U. (1994) Misspecification of long memory seasonal time series, Journal of Time Series Analysis, 15, pp. 19-30.

Hasza, D.P. \& Fuller, W.A. (1982) Testing for nonstationary parameter specifications in seasonal time series models, Annals of Statistics, 10, pp.1209-1216.

Hylleberg, S., Engle, R.F., Granger, C.W.J. \& Yoo, B.S. (1990) Seasonal integration and cointegration, Journal of Econometrics, 44, pp. 215-238.

Nelson, C.R. \& Plosser, C.I. (1982) Trends and random walks in macroeconomic time series' Journal of Monetary Economics, 10, pp. 139-162.

Ooms, M. (1995) Flexible seasonal long memory and economic time series, Discussion Paper 9951/A. Presented at the World Congress Econometric Society 1995, Tokyo, Econometric Institute, Erasmus University Rotterdam.

Press, W.H., Flannery, B.P., Teukolsky, S.A. \& Vetterling, W.T. (1986) Numerical recipes. The art of scientific computing, Cambridge University Press, Cambridge.

Robinson, P.M. (1994) Efficient tests of nonstationary hypotheses, Journal of the American Statistical Association, 89, pp. 1420-1437. 
Robinson, P.M. (1995a) Log-periodogram regression of time series with long range dependence, Annals of Statistics, 23, pp.1048-1072.

Robinson P.M. (1995b) Gaussian semi-parametric estimation of long-range dependence, Annals of Statistics, 23, pp. 1630-1661. 


\begin{tabular}{|c|c|c|c|c|c|}
\hline \multicolumn{7}{|c|}{ TABLE 1 } \\
\hline \multicolumn{7}{|c|}{ Finite-sample critical values of $\hat{R}$ in (3) in a model given by (1) } \\
\hline Percentiles & $\mathrm{T}=48$ & $\mathrm{~T}=96$ & $\mathrm{~T}=120$ & $\mathrm{~T}=240$ & $\chi_{3}^{2}$ \\
\hline $20 \%$ & 7.79 & 6.64 & 6.14 & 5.33 & 4.64 \\
\hline $10 \%$ & 9.64 & 8.46 & 8.12 & 7.17 & 6.25 \\
\hline $5 \%$ & 11.45 & 10.36 & 9.94 & 8.45 & 7.81 \\
\hline $2.5 \%$ & 13.34 & 12.26 & 11.96 & 10.77 & 9.35 \\
\hline $2 \%$ & 14.01 & 12.88 & 12.69 & 11.54 & 11.30 \\
\hline $1 \%$ & 16.01 & 14.91 & 14.86 & 13.64 & 12.80 \\
\hline $0.5 \%$ & 18.01 & 17.87 & 16.58 & 15.73 & 16.30 \\
\hline $0.1 \%$ & 24.68 & 23.12 & 24.69 & 21.17 & 17.60 \\
\hline
\end{tabular}

50,000 replications were used in each case. 


\begin{tabular}{|c|c|c|c|c|c|c|}
\hline \multicolumn{7}{|c|}{ TABLE 2} \\
\hline \multicolumn{7}{|c|}{ Rejection frequencies of $\hat{R}$ in (3) against (5) } \\
\hline \multicolumn{7}{|c|}{ True model: $(1-L)\left(1-L^{4}\right)\left(1-L^{12}\right) y_{t}=u_{t} ; \quad u_{t}$ is white noise. } \\
\hline \multicolumn{7}{|c|}{ Alternatives: $(1-L)^{1+\theta_{1}}\left(1-L^{4}\right)^{1+\theta_{2}}\left(1-L^{12}\right)^{1+\theta_{3}} y_{t}=u_{t}$} \\
\hline$\theta_{1}$ & $\theta_{2}$ & $\theta_{3}$ & $\mathrm{~T}=48$ & $\mathrm{~T}=96$ & $\mathrm{~T}=120$ & $\mathrm{~T}=240$ \\
\hline-1.00 & -1.00 & -1.00 & $.806(.922)$ & $.993(.995)$ & $.997(.998)$ & $1.000(1.000)$ \\
\hline-1.00 & -1.00 & -0.50 & $.802(.892)$ & $.990(.995)$ & $.996(.998)$ & $1.000(1.000)$ \\
\hline-1.00 & -1.00 & 0.00 & $.705(.827)$ & $.974(.985)$ & $.989(.993)$ & $.999(.999)$ \\
\hline-1.00 & -0.50 & -1.00 & $.757(.866)$ & $.987(.992)$ & $.995(.997)$ & $1.000(1.000)$ \\
\hline-1.00 & -0.50 & -0.50 & $.641(.778)$ & $.965(.977)$ & $.985(.990)$ & $.999(.999)$ \\
\hline-1.00 & -0.50 & 0.00 & $.577(.695)$ & $.915(.944$ & $.957(.972)$ & $.998(.998)$ \\
\hline-1.00 & 0.00 & -1.00 & $.610(.764)$ & $.960(.976)$ & $.983(.989)$ & $.999(.999)$ \\
\hline-1.00 & 0.00 & -0.50 & $.548(.664)$ & $.898(.930)$ & $.946(.962)$ & $.997(.998)$ \\
\hline-1.00 & 0.00 & 0.00 & $.598(.679)$ & $.864(.901)$ & $.914(.936)$ & $.991(.992)$ \\
\hline-0.50 & -1.00 & -1.00 & $.477(.625)$ & $.869(.901)$ & $.936(.954)$ & $.999(.999)$ \\
\hline-0.50 & -1.00 & -0.50 & $.400(.560)$ & $.856(.892)$ & $.925(.944)$ & $.997(.997)$ \\
\hline-0.50 & -1.00 & 0.00 & $.372(.501)$ & $.828(.873)$ & $.908(.932)$ & $.995(.996)$ \\
\hline-0.50 & -0.50 & -1.00 & $.368(.534)$ & $.836(.873)$ & $.906(.925)$ & $.992(.993)$ \\
\hline-0.50 & -0.50 & -0.50 & $.341(.469)$ & $.797(.844)$ & $.884(.911)$ & $.992(.993)$ \\
\hline-0.50 & -0.50 & 0.00 & $.412(.514)$ & $.799(.845)$ & $.876(.904)$ & $.987(.988)$ \\
\hline-0.50 & 0.00 & -1.00 & $.536(.671)$ & $.902(.939)$ & $.950(.969)$ & $\begin{array}{l}.999(.999) \\
\end{array}$ \\
\hline-0.50 & 0.00 & -0.50 & $.505(.627)$ & $.828(.874)$ & $.892(.922)$ & $.989(.991)$ \\
\hline-0.50 & 0.00 & 0.00 & $.563(.680)$ & $.903(.934)$ & $.951(.966)$ & $.998(.998)$ \\
\hline 0.00 & -1.00 & -1.00 & $.303(.421)$ & $.590(.695)$ & $.759(.831)$ & $.999(1.000)$ \\
\hline 0.00 & -1.00 & -0.50 & $.323(.452)$ & $.573(.676)$ & $.716(.796)$ & $.995(.997)$ \\
\hline 0.00 & -1.00 & 0.00 & $.296(.429)$ & $.532(.653)$ & $.692(.781)$ & $.989(.990)$ \\
\hline 0.00 & -0.50 & -1.00 & $.385(.520)$ & $.479(.602)$ & $.585(.676)$ & $.935(.944)$ \\
\hline 0.00 & -0.50 & -0.50 & $.275(.428)$ & $.393(.506)$ & $.503(.596)$ & $.915(.925)$ \\
\hline 0.00 & -0.50 & 0.00 & $.119(.224)$ & $.316(.417)$ & $.450(.547)$ & $.967(.973)$ \\
\hline 0.00 & 0.00 & -1.00 & $.880(.957)$ & $.986(.993)$ & $.990(.994)$ & $.999(.999)$ \\
\hline 0.00 & 0.00 & -0.50 & $.608(.807)$ & $.878(.931)$ & $.922(.950)$ & $.991(.992)$ \\
\hline 0.00 & 0.00 & 0.00 & $.050(.199)$ & $.050(.127)$ & $.050(.110)$ & $.050(.079)$ \\
\hline
\end{tabular}

50,000 replications were used in each case. In parentheses, the rejection frequencies based on the asymptotic critical values. 


\section{TABLE 3}

Testing $\mathrm{H}_{\mathrm{o}}(2)$ in (1) with $\hat{R}$ given by (3) with white noise $\mathrm{u}_{\mathrm{t}}$

\begin{tabular}{|c|c|c|c|c|c|c|}
\hline \multicolumn{2}{|c|}{ Series: U.S. M1 } & \multicolumn{5}{|c|}{$\mathrm{d}_{3}$} \\
\hline $\mathrm{d}_{1}$ & $\mathrm{~d}_{2}$ & 0.00 & 0.25 & 0.50 & 0.75 & 1.00 \\
\hline 0.00 & 0.00 & 136.76 & 148.34 & 147.44 & 150.66 & 361.77 \\
\hline 0.00 & 0.25 & 148.29 & 145.43 & 149.41 & 334.46 & 443.64 \\
\hline 0.00 & 0.50 & 139.08 & 143.74 & 292.52 & 361.45 & 337.19 \\
\hline 0.00 & 0.75 & 124.41 & 233.58 & 273.30 & 256.29 & 235.01 \\
\hline 0.00 & 1.00 & 161.86 & 190.08 & 184.81 & 174.97 & 167.03 \\
\hline 0.25 & 0.00 & 147.72 & 142.81 & 139.94 & 286.32 & 357.38 \\
\hline 0.25 & 0.25 & 133.99 & 130.99 & 240.34 & 279.17 & 253.06 \\
\hline 0.25 & 0.50 & 105.86 & 181.52 & 201.48 & 184.75 & 167.46 \\
\hline 0.25 & 0.75 & 119.41 & 134.59 & 129.30 & 121.66 & 115.75 \\
\hline 0.25 & 1.00 & 93.70 & 90.70 & 89.51 & 87.84 & 86.52 \\
\hline 0.50 & 0.00 & 120.94 & 103.49 & 159.27 & 168.31 & 145.70 \\
\hline 0.50 & 0.25 & 75.43 & 112.20 & 115.48 & 102.34 & 91.13 \\
\hline 0.50 & 0.50 & 73.14 & 75.04 & 70.80 & 66.15 & 62.78 \\
\hline 0.50 & 0.75 & 62.03 & 52.18 & 51.06 & 50.15 & 49.50 \\
\hline 0.50 & 1.00 & 58.69 & 44.35 & 43.86 & 43.94 & 44.06 \\
\hline 0.75 & 0.00 & 30.63 & 39.79 & 38.26 & 32.83 & 29.03 \\
\hline 0.75 & 0.25 & 32.71 & 25.68 & 24.28 & 23.29 & 22.72 \\
\hline 0.75 & 0.50 & 39.23 & 22.87 & 22.42 & 22.66 & 22.89 \\
\hline 0.75 & 0.75 & 46.97 & 25.82 & 25.14 & 25.53 & 25.85 \\
\hline 0.75 & 1.00 & 53.62 & 29.90 & 28.99 & 29.40 & 29.73 \\
\hline 1.00 & 0.00 & 9.16 & $2.36 *$ & $4.91 *$ & $6.95 *$ & 9.48 \\
\hline 1.00 & 0.25 & 26.58 & 9.33 & 10.70 & 12.38 & 13.60 \\
\hline 1.00 & 0.50 & 42.62 & 17.84 & 17.91 & 19.02 & 19.80 \\
\hline 1.00 & 0.75 & 54.17 & 25.41 & 24.73 & 25.48 & 26.01 \\
\hline 1.00 & 1.00 & 62.23 & 31.56 & 30.57 & 31.23 & 31.70 \\
\hline
\end{tabular}

$*$ and in bold: Non-rejection values at the $95 \%$ significance level. 
TABLE 4

Testing $\mathrm{H}_{\mathrm{o}}(2)$ in (1) with $\hat{R}$ given by (3) with white noise $\mathrm{u}_{\mathrm{t}}$

\begin{tabular}{|c|c|c|c|c|c|c|}
\hline \multicolumn{2}{|c|}{ Series: U.S. M2 } & \multicolumn{5}{|c|}{$\mathrm{d}_{3}$} \\
\hline $\mathrm{d}_{1}$ & $\mathrm{~d}_{2}$ & 0.00 & 0.25 & 0.50 & 0.75 & 1.00 \\
\hline 0.00 & 0.00 & 141.50 & 157.02 & 150.53 & 147.81 & 351.08 \\
\hline 0.00 & 0.25 & 156.72 & 148.06 & 148.63 & 328.65 & 429.95 \\
\hline 0.00 & 0.50 & 142.25 & 145.84 & 290.83 & 349.84 & 317.18 \\
\hline 0.00 & 0.75 & 130.97 & 235.69 & 265.08 & 241.77 & 219.86 \\
\hline 0.00 & 1.00 & 167.82 & 186.71 & 176.44 & 166.24 & 160.41 \\
\hline 0.25 & 0.00 & 156.21 & 145.54 & 140.65 & 282.81 & 344.37 \\
\hline 0.25 & 0.25 & 138.15 & 134.67 & 240.02 & 268.59 & 235.81 \\
\hline 0.25 & 0.50 & 114.52 & 184.00 & 194.13 & 172.85 & 155.85 \\
\hline 0.25 & 0.75 & 123.62 & 131.24 & 122.48 & 115.18 & 111.28 \\
\hline 0.25 & 1.00 & 91.45 & 87.93 & 86.61 & 86.12 & 86.36 \\
\hline 0.50 & 0.00 & 127.24 & 108.64 & 159.70 & $1^{\wedge} 59.79$ & 133.22 \\
\hline 0.50 & 0.25 & 84.87 & 114.14 & 109.68 & 94.13 & 83.80 \\
\hline 0.50 & 0.50 & 72.76 & 71.87 & 66.04 & 62.11 & 60.27 \\
\hline 0.50 & 0.75 & 54.48 & 49.56 & 49.03 & 49.06 & 49.42 \\
\hline 0.50 & 1.00 & 49.39 & 43.00 & 43.39 & 44.06 & 44.73 \\
\hline 0.75 & 0.00 & 38.87 & 41.31 & 35.23 & 29.06 & 26.01 \\
\hline 0.75 & 0.25 & 26.72 & 23.57 & 22.09 & 21.64 & 21.80 \\
\hline 0.75 & 0.50 & 26.32 & 21.05 & 21.51 & 22.16 & 22.75 \\
\hline 0.75 & 0.75 & 31.80 & 24.41 & 24.74 & 25.30 & 25.77 \\
\hline 0.75 & 1.00 & 37.38 & 28.56 & 28.65 & 29.10 & 29.49 \\
\hline 1.00 & 0.00 & $1.61 *$ & $2.58 *$ & $5.24 *$ & 8.99 & 9.82 \\
\hline 1.00 & 0.25 & 12.29 & 9.23 & 11.18 & 12.66 & 13.71 \\
\hline 1.00 & 0.50 & 24.06 & 17.26 & 18.10 & 18.93 & 19.53 \\
\hline 1.00 & 0.75 & 33.18 & 24.25 & 24.52 & 25.04 & 25.43 \\
\hline 1.00 & 1.00 & 39.88 & 29.93 & 30.06 & 30.54 & 30.93 \\
\hline
\end{tabular}

$*$ and in bold: Non-rejection values at the $5 \%$ significance level. 


\section{TABLE 5}

Testing $\mathrm{H}_{\mathrm{o}}(2)$ in (1) with $\hat{R}$ given by (3) with white noise $\mathrm{u}_{\mathrm{t}}$

\begin{tabular}{|c|c|c|c|c|c|c|}
\hline \multicolumn{2}{|c|}{ Series: U.S. M3 } & \multicolumn{5}{|c|}{$\mathrm{d}_{3}$} \\
\hline $\mathrm{d}_{1}$ & $\mathrm{~d}_{2}$ & 0.00 & 0.25 & 0.50 & 0.75 & 1.00 \\
\hline 0.00 & 0.00 & 140.57 & 153.08 & 145.36 & 153.43 & 365.92 \\
\hline 0.00 & 0.25 & 152.72 & 143.52 & 155.72 & 347.07 & 457.80 \\
\hline 0.00 & 0.50 & 139.24 & 155.08 & 312.73 & 375.79 & 331.13 \\
\hline 0.00 & 0.75 & 143.45 & 259.29 & 287.58 & 253.30 & 223.34 \\
\hline 0.00 & 1.00 & 188.76 & 204.61 & 186.10 & 170.18 & 161.44 \\
\hline 0.25 & 0.00 & 152.32 & 141.67 & 149.91 & 306.27 & 373.39 \\
\hline 0.25 & 0.25 & 136.26 & 146.71 & 266.46 & 295.18 & 249.45 \\
\hline 0.25 & 0.50 & 130.26 & 210.82 & 216.92 & 184.61 & 160.27 \\
\hline 0.25 & 0.75 & 145.79 & 149.30 & 132.70 & 120.20 & 113.51 \\
\hline 0.25 & 1.00 & 103.81 & 96.75 & 91.99 & 89.34 & 88.26 \\
\hline 0.50 & 0.00 & 127.66 & 123.93 & 187.27 & 182.38 & 143.93 \\
\hline 0.50 & 0.25 & 103.11 & 139.81 & 128.57 & 103.66 & 87.81 \\
\hline 0.50 & 0.50 & 92.03 & 86.68 & 74.55 & 66.69 & 62.70 \\
\hline 0.50 & 0.75 & 64.07 & 56.96 & 53.81 & 52.13 & 51.40 \\
\hline 0.50 & 1.00 & 54.22 & 47.56 & 46.67 & 46.36 & 46.32 \\
\hline 0.75 & 0.00 & 55.07 & 57.84 & 45.28 & 33.78 & 28.05 \\
\hline 0.75 & 0.25 & 37.85 & 31.56 & 26.53 & 24.11 & 23.22 \\
\hline 0.75 & 0.50 & 30.78 & 25.08 & 24.16 & 23.90 & 23.91 \\
\hline 0.75 & 0.75 & 33.50 & 26.94 & 26.56 & 26.56 & 26.64 \\
\hline 0.75 & 1.00 & 37.72 & 30.23 & 29.88 & 29.94 & 30.05 \\
\hline 1.00 & 0.00 & 2.96* & $3.46 *$ & $5.43 *$ & 8.87 & 9.86 \\
\hline 1.00 & 0.25 & 11.63 & 9.62 & 11.35 & 12.75 & 13.77 \\
\hline 1.00 & 0.50 & 22.63 & 17.56 & 18.25 & 18.96 & 19.52 \\
\hline 1.00 & 0.75 & 3.23 & 24.37 & 24.53 & 24.94 & 25.30 \\
\hline 1.00 & 1.00 & 37.48 & 29.86 & 29.92 & 30.32 & 30.68 \\
\hline
\end{tabular}

\footnotetext{
$*$ and in bold: Non-rejection values at the $5 \%$ significance level.
} 


\section{TABLE 6}

Testing $\mathrm{H}_{\mathrm{o}}(2)$ in (1) with $\hat{R}$ given by (3) with white noise $\mathrm{u}_{\mathrm{t}}$

\begin{tabular}{|c|c|c|c|c|c|c|}
\hline \multicolumn{2}{|c|}{ Series: U.S. MZM } & \multicolumn{5}{|c|}{$d_{3}$} \\
\hline $\mathrm{d}_{1}$ & $\mathrm{~d}_{2}$ & 0.00 & 0.25 & 0.50 & 0.75 & 1.00 \\
\hline 0.00 & 0.00 & 135.88 & 139.21 & 143.11 & 137.55 & 243.57 \\
\hline 0.00 & 0.25 & 139.41 & 142.24 & 138.24 & 242.93 & 407.10 \\
\hline 0.00 & 0.50 & 139.73 & 137.27 & 233.14 & 348.19 & 342.95 \\
\hline 0.00 & 0.75 & 127.57 & 208.78 & 277.87 & 264.40 & 235.63 \\
\hline 0.00 & 1.00 & 163.37 & 204.44 & 194.49 & 177.85 & 167.52 \\
\hline 0.25 & 0.00 & 139.45 & 141.42 & 135.44 & 223.76 & 344.82 \\
\hline 0.25 & 0.25 & 138.08 & 132.95 & 208.29 & 283.51 & 265.47 \\
\hline 0.25 & 0.50 & 119.36 & 178.20 & 215.98 & 196.46 & 171.96 \\
\hline 0.25 & 0.75 & 131.14 & 151.74 & 139.69 & 126.44 & 118.95 \\
\hline 0.25 & 1.00 & 106.82 & 99.42 & 94.38 & 91.71 & 91.03 \\
\hline 0.50 & 0.00 & 132.64 & 119.89 & 160.09 & 187.98 & 162.67 \\
\hline 0.50 & 0.25 & 101.47 & 128.53 & 135.49 & 115.26 & 98.13 \\
\hline 0.50 & 0.50 & 87.98 & 90.86 & 80.00 & 71.52 & 67.28 \\
\hline 0.50 & 0.75 & 67.64 & 58.19 & 55.12 & 53.85 & 53.71 \\
\hline 0.50 & 1.00 & 58.78 & 47.12 & 46.69 & 47.11 & 47.75 \\
\hline 0.75 & 0.00 & 63.96 & 62.16 & 53.77 & 41.49 & 34.17 \\
\hline 0.75 & 0.25 & 40.75 & 35.27 & 29.67 & 26.72 & 25.72 \\
\hline 0.75 & 0.50 & 35.05 & 25.35 & 24.57 & 24.69 & 25.06 \\
\hline 0.75 & 0.75 & 38.77 & 26.37 & 26.34 & 26.83 & 27.26 \\
\hline 0.75 & 1.00 & 44.08 & 29.71 & 29.58 & 30.05 & 30.42 \\
\hline 1.00 & 0.00 & $5.14 *$ & 4.32* & 5.79* & 8.32 & 9.63 \\
\hline 1.00 & 0.25 & 15.87 & 9.20 & 10.92 & 12.34 & 13.30 \\
\hline 1.00 & 0.50 & 28.55 & 16.92 & 17.64 & 18.45 & 18.95 \\
\hline 1.00 & 0.75 & 38.47 & 23.86 & 23.94 & 24.41 & 24.69 \\
\hline 1.00 & 1.00 & 45.63 & 29.49 & 29.36 & 29.79 & 30.06 \\
\hline
\end{tabular}

* and in bold: Non-rejection values at the $5 \%$ significance level. 
TABLE 7

Testing $\mathrm{H}_{\mathrm{o}}(2)$ in (1) with $\hat{R}$ given by (3) with $\operatorname{AR}(1) \mathrm{u}_{\mathrm{t}}$

\begin{tabular}{|c|c|c|c|c|c|c|}
\hline \multicolumn{2}{|c|}{ Series: U.S. M2 } & \multicolumn{5}{|c|}{$\mathrm{d}_{3}$} \\
\hline $\mathrm{d}_{1}$ & $\mathrm{~d}_{2}$ & 0.00 & 0.25 & 0.50 & 0.75 & 1.00 \\
\hline 0.00 & 0.00 & $1.13 *$ & $2.17 *$ & $2.55 *$ & $7.22 *$ & 8.51 \\
\hline 0.00 & 0.25 & 16.55 & 9.39 & 12.30 & 11.91 & 12.58 \\
\hline 0.00 & 0.50 & 30.10 & 18.95 & 16.41 & 16.86 & 18.05 \\
\hline 0.00 & 0.75 & 34.91 & 20.84 & 21.97 & 23.11 & 23.78 \\
\hline 0.00 & 1.00 & 33.12 & 27.71 & 28.42 & 28.85 & 29.27 \\
\hline 0.25 & 0.00 & 20.44 & 20.24 & 25.32 & 18.55 & 11.72 \\
\hline 0.25 & 0.25 & 36.52 & 32.20 & 19.18 & 12.66 & 13.42 \\
\hline 0.25 & 0.50 & 51.32 & 19.58 & 15.44 & 16.76 & 18.45 \\
\hline 0.25 & 0.75 & 28.48 & 20.04 & 21.18 & 22.69 & 23.91 \\
\hline 0.25 & 1.00 & 33.99 & 26.04 & 27.09 & 28.25 & 29.06 \\
\hline 0.50 & 0.00 & 52.56 & 73.48 & 22.03 & 8.08 & 9.31 \\
\hline 0.50 & 0.25 & 83.30 & 13.56 & 10.12 & 11.90 & 14.13 \\
\hline 0.50 & 0.50 & 20.15 & 14.72 & 16.45 & 18.61 & 20.35 \\
\hline 0.50 & 0.75 & 28.80 & 22.16 & 23.98 & 25.59 & 26.65 \\
\hline 0.50 & 1.00 & 37.29 & 29.52 & 30.80 & 31.91 & 32.60 \\
\hline 0.75 & 0.00 & 46.25 & $4.65^{*}$ & $7.22 *$ & 8.00 & 9.36 \\
\hline 0.75 & 0.25 & 11.10 & 8.92 & 10.91 & 13.02 & 14.70 \\
\hline 0.75 & 0.50 & 21.79 & 16.80 & 18.83 & 20.47 & 21.54 \\
\hline 0.75 & 0.75 & 32.34 & 25.51 & 26.82 & 27.87 & 28.54 \\
\hline 0.75 & 1.00 & 40.65 & 32.72 & 33.70 & 34.56 & 35.12 \\
\hline 1.00 & 0.00 & $1.57 *$ & $2.66 *$ & $5.79 *$ & 8.44 & 10.42 \\
\hline 1.00 & 0.25 & 10.42 & 12.71 & 10.74 & 13.53 & 15.52 \\
\hline 1.00 & 0.50 & 25.73 & 20.56 & 22.15 & 23.33 & 24.11 \\
\hline 1.00 & 0.75 & 35.96 & 28.90 & 29.90 & 30.74 & 31.29 \\
\hline 1.00 & 1.00 & 43.44 & 35.60 & 36.51 & 37.35 & 37.92 \\
\hline
\end{tabular}

$*$ and in bold: Non-rejection values at the 5\% significance level. 
TABLE 8

Testing $\mathrm{H}_{\mathrm{o}}(2)$ in (1) with $\hat{R}$ given by (3) with monthly seasonal $\operatorname{AR}(1) \mathrm{u}_{\mathrm{t}}$

\begin{tabular}{|c|c|c|c|c|c|c|}
\hline \multicolumn{2}{|c|}{ Series: U.S. M2 } & \multicolumn{5}{|c|}{$\mathrm{d}_{3}$} \\
\hline $\mathrm{d}_{1}$ & $\mathrm{~d}_{2}$ & 0.00 & 0.25 & 0.50 & 0.75 & 1.00 \\
\hline 0.00 & 0.00 & 565.70 & 731.75 & 450.34 & 303.12 & 355.63 \\
\hline 0.00 & 0.25 & 842.15 & 355.95 & 258.63 & 324.80 & 489.66 \\
\hline 0.00 & 0.50 & 263.73 & 215.61 & 285.89 & 411.10 & 431.37 \\
\hline 0.00 & 0.75 & 176.10 & 233.21 & 306.84 & 308.67 & 280.97 \\
\hline 0.00 & 1.00 & 166.82 & 200.04 & 200.03 & 187.55 & 177.10 \\
\hline 0.25 & 0.00 & 718.59 & 22855 & 194.60 & 272.31 & 416.61 \\
\hline 0.25 & 0.25 & 181.19 & 163.79 & 234.87 & 334.36 & 339.44 \\
\hline 0.25 & 0.50 & 133.41 & 182.68 & 233.41 & 227.58 & 203.33 \\
\hline 0.25 & 0.75 & 121.85 & 142.05 & 139.47 & 129.76 & 122.32 \\
\hline 0.25 & 1.00 & 92.05 & 89.95 & 87.68 & 85.95 & 85.45 \\
\hline 0.50 & 0.00 & 83.97 & 99.69 & 160.71 & 221.03 & 211.10 \\
\hline 0.50 & 0.25 & 81.79 & 116.27 & 141.00 & 130.52 & 112.81 \\
\hline 0.50 & 0.50 & 71.03 & 79.34 & 75.81 & 69.67 & 65.49 \\
\hline 0.50 & 0.75 & 55.05 & 51.12 & 49.51 & 48.61 & 48.45 \\
\hline 0.50 & 1.00 & 50.00 & 43.88 & 42.90 & 42.81 & 43.09 \\
\hline 0.75 & 0.00 & 32.08 & 44.78 & 49.64 & 42.01 & 34.13 \\
\hline 0.75 & 70.25 & 25.88 & 26.29 & 24.11 & 21.99 & 20.97 \\
\hline 0.75 & 0.50 & 26.99 & 21.98 & 2114 & 21.01 & 21.19 \\
\hline 0.75 & 0.75 & 32.33 & 25.94 & 25.05 & 24.93 & 24.99 \\
\hline 0.75 & 1.00 & 37.23 & 30.40 & 29.36 & 29.15 & 29.10 \\
\hline 1.00 & 0.00 & $1.43 *$ & $1.07 *$ & $1.92 *$ & 3.00* & 4.15* \\
\hline 1.00 & 0.25 & 12.84 & 8.63 & 8.94 & 9.73 & 10.48 \\
\hline 1.00 & 0.50 & 24.74 & 18.53 & 17.98 & 18.09 & 18.25 \\
\hline 1.00 & 0.75 & 33.10 & 26.29 & 25.40 & 25.25 & 25.19 \\
\hline 1.00 & 1.00 & 39.01 & 32.00 & 31.03 & 30.88 & 30.83 \\
\hline
\end{tabular}

* and in bold: Non-rejection values at the 5\% significance level. 